\title{
La medición de la Orientación Emprendedora en las empresas familiares: una revisión crítica de la literatura
}

\section{The measurement of the Entrepreneurial Orientation in family firms: a critical review of the literature}

\author{
Unai Arzubiaga Orueta ${ }^{\mathrm{a},}{ }^{*} \cdot$ Txomin Iturralde Jainaga $^{\mathrm{b}} \cdot$ Amaia Maseda García $^{\mathrm{c}}$ \\ ${ }^{a, b, c}$ Facultad de Ciencias Económicas y Empresariales de la Universidad del País Vasco, UPV/EHU. Avenida \\ Lehendakari Agirre, 83, 48015 (Bilbao)
}

D A T O S A R T ÍC U L O

Historial:

Recibido 01-10-2012

Aceptado 16-11-2012

Palabras clave:

Orientación emprendedora

Empresa Familiar

Dimensiones

Escala de medición

Códigos JEL:

L10, M20

A R T I C L E I N F O

Article history:

Received 01-10-2012

Accepted 16-11-2012

Keywords:

Entrepreneurial orientation

Family firm

Dimensions

Measurement scale

JEL codes:

L10, M20

\begin{abstract}
R E S U M E N
El presente trabajo realiza una profunda revisión del constructo Orientación Emprendedora (EO) analizando el caso específico de las empresas familiares. Se ha llevado a cabo una clasificación de las obras que contienen un modelo donde el EO desempeña dos funciones diferentes: por un lado, trabajos en los que éste constructo se ha tomado como una variable dependiente y, por otra parte, artículos en donde ha sido entendido como una variable independiente. Con el propósito de analizar en profundidad el constructo EO, se ha llevado a cabo una revisión de la literatura de cada una de las cinco dimensiones, centrándose en el caso específico de las empresas familiares. Unido a estas dimensiones, se ha realizado un análisis de cómo se ha medido el constructo $\mathrm{EO}$ en la literatura científica, centrándose en dos de las tendencias más aceptadas: la medición a través de una escala de tres dimensiones o hacerlo mediante una escala de cinco. Finalmente, hemos llevado a cabo una reflexión sobre cuál de estas escalas es más adecuada para el caso específico de las empresas familiares justificando nuestra posición.
\end{abstract}

\begin{abstract}
A B S T R A C T
The present paper makes a deep review of the Entrepreneurial Orientation construct analyzing the specific case of family firms. A classification of the works that contain a model where EO plays two different roles has been done: on the one hand, works where this construct has been taken as a dependent variable and, on the other hand, papers where it has been understood as an independent variable. With the purpose of analizying the EO construct in a great depth, a literature review of each five dimensions has been done, focusing on the specific case of family firms. Joining to these dimensions, it has been done an examination of how the EO construct has been measured, focusing on two of the most accepted trends: measuring it with a three dimension scale or doing it with a five dimension one. Finally, we made a reflection about which of these scales is more appropriate for the specific case of family firms justifying our position.
\end{abstract}

\footnotetext{
* Autor de contacto.
}

Correoselectrónicos: unai.arzubiaga@ehu.es, txomin.iturralde@ehu.es,amaia.maseda@ehu.es

Financiación: Cátedra de Empresa Familiar de la UPV/EHU (DFB/BFA y el Fondo Social Europeo). UPV/EHU (Proyecto UPV/EHU 10/30). 


\section{Introducción}

El Emprendimiento Corporativo ha sido reconocido como un medio viable para promover y sostener el desempeño de la empresa (Schöllhammer,1982; Burgelman, 1984; Kanter, 1982; Guth y Ginsberg 1990; Zahra 1991). Sus objetivos principales son la renovación estratégica de la organización (Guth y Ginsberg, 1990), la obtención de conocimientos para lograr nuevas fuentes de ingresos en el futuro, alcanzar el éxito en un contexto internacional (Birkinshaw, 1997) basándose para ello en la eficacia a la hora de configurar los recursos con el fin último de obtener ventajas competitivas (Covin y Miles, 1999; Covin et al, 2006), la obtención de rentabilidad (Zahra, 1991) y el desarrollo de innovaciones.

Los comportamientos emprendedores pueden ser especialmente cruciales para una empresa familiar (Astrachan, 2003). La literatura señala que las empresas familiares son muy diferentes a otras empresas debido a las características únicas de los miembros individuales de la familia, el sistema familiar y el sistema de negocio. A pesar de estas especificidades y del gran potencial del emprendimiento para lograr la subsistencia de las empresas familiares a lo largo de distintas generaciones, se ha investigado poco acerca del emprendimiento corporativo en este tipo de empresas. Por ello, uno de los principales retos de la literatura científica en esta área radica en estudiar la orientación emprendedora (EO) en la empresa familiar. La relación entre la orientación emprendedora de la empresa y su desempeño ha sido ampliamente estudiada conceptualmente (Covin y Slevin, 1991; Lumpkin y Dess, 1996) y empíricamente (Wiklund y Shepherd, 2005; Covin y Slevin, 1989; Lumpkin y Dess, 2001). Sin embargo, muchas preguntas siguen aún sin respuesta (Moreno y Casillas, 2008).

Para medir la relación entre la orientación emprendedora y el rendimiento, existe un constructo multidimensional denominado Orientación Emprendedora (EO) por Miller (1983), la cual permite a los autores comparar el comportamiento emprendedor entre distintas empresas o, incluso, comparar este comportamiento en una misma empresa en diferentes espacios de tiempo. A tal efecto, la mayoría de los autores han definido EO de una manera similar, es decir, como un fenómeno organizacional relacionado con los procesos, los métodos y las actividades de toma de decisiones de la empresa (por ejemplo, Covin y Slevin, 1989; Hughes y Morgan, 2007; Jantunen, et al, 2005;. Lumpkin y Dess, 1996; Rauch, et al, 2009;. Wiklund y Shepherd, 2005). Miller (1983), que fue un pionero en el uso del constructo EO, lo consideró como un constructo multidimensional compuesto por tres dimensiones: la innovación, la asunción de riesgos y la proactividad, teniendo que covariar positivamente entre ellos (Covin y Wales, 2001). Por su parte, Lumpkin y Dess (1996) extendieron el dominio del constructo EO añadiendo dos dimensiones adicionales: la autonomía y la agresividad competitiva.

Sin embargo, debido a lo necesario que resulta acumular nuevos conocimientos en torno a este concepto, la mayoría de los autores han definido EO de la manera anteriormente mencionada.

En este trabajo, realizamos una revisión de la literatura del concepto de orientación emprendedora (EO) en general, y más concretamente, en las empresas familiares. Para ello nos hemos centrado en sus cinco dimensiones, haciendo una revisión exhaustiva de cada una de ellas y centrándonos en el caso específico de las empresas familiares. Además, aparte de presentar y analizar dos de las principales conceptualizaciones del constructo $\mathrm{EO}$, hemos concluido sobre el uso de ambos.

El documento está estructurado de la siguiente manera: después de una introducción, se ha procedido a realizar una revisión de la literatura del concepto de EO tanto a nivel general como en el caso específico de las empresas familiares. Posteriormente, se ha llevado a cabo un estudio de las cinco dimensiones de EO, centrándonos en el caso específico de las empresas familiares. En la siguiente sección, hemos examinado algunos de los otros aspectos que pueden tener efectos en el EO antes de llevar a cabo una reflexión sobre la utilización en el futuro de este concepto. Este 
artículo finaliza con las consabidas referencias bibliográficas.

\section{Orientación conceptualización}

Emprendedora:

Los orígenes del constructo EO están ligados al concepto de Emprendimiento Corporativo. El Corporate Entrepreneurship puede albergar dos formas diferentes: como una iniciativa empresarial de capital riesgo o como un emprendimiento estratégico (Morris, et al 2010). Por un lado, una iniciativa empresarial de capital riesgo supone, entre otros, una entrada en un mercado nuevo implicando, por lo general, la creación de una nueva empresa.

Por otro lado, el emprendimiento estratégico puede ser entendido simultáneamente en términos de comportamientos de búsqueda de oportunidades y búsqueda de ventajas (Ireland et al., 2003) con el fin último de crear una ventaja competitiva (Kuratko, 2010). Algunos de los principales factores clave para ello son la capacidad de las empresas para innovar, iniciar el cambio y reaccionar rápidamente para cambiar con flexibilidad y destreza (Naman y Slevin 1993), siendo estas características las que distinguen a una empresa emprendedora de otra que no lo es. Sin embargo, no resulta sencilla la medición del grado de emprendimiento de una compañía. Miller (1983) ideó un constructo denominado Orientación Emprendedora, refiriéndose esta a la forma de pensar de las compañías involucradas en un afán de crear otras empresas y establecer un marco adecuado para la investigación de la actividad emprendedora (Naldi et al., 2007). Este autor entendió el EO como un constructo compuesto por tres subdimensiones -innovación, asunción de riesgos, y proactividad- que deben covariar positivamente para que el EO se manifieste (Covin y Wales, 2011).

Más tarde, Covin y Slevin (1989) completaron la definición de Miller afirmando que "la orientación emprendedora de una empresa se demuestra por el grado en que la alta dirección está dispuesta a asumir los riesgos relacionados con la empresa (dimensión de asunción de riesgos), para favorecer el cambio y la innovación con el fin de obtener una ventaja competitiva para su empresa (dimensión de innovación), y competir agresivamente con otras firmas (dimensión de proactividad) ".

A pesar de que un gran número de autores han adoptado definiciones y concepciones similares a las de Miller (1983) y Covin y Slevin (1989), a mediados de los noventa, Lumpkin y Dess (1996) extendieron el dominio del constructo EO. Para ello, incluyeron dos dimensiones adicionales: la agresividad competitiva y la autonomía. Dichos autores afirmaron que estas cinco dimensiones son complementarias entre sí y definieron EO como "los procesos, las prácticas y las actividades de toma de decisiones que llevan a una nueva entrada (en el mercado)" (Lumpkin y Dess, 1996). Esta definición de EO es diferente a la Covin y Slevin (1989). Así, mientras esta última se refiere a EO como una postura estratégica que refleja las decisiones y los procesos de las empresas, la definición de EO de Lumpkin y Dess (1996) los limita a aquellas que conducen a una nueva entrada (George y Marino, 2011).

Basándose en estas dos definiciones tan ampliamente aceptadas, en los últimos años han proliferado los estudios empíricos y trabajos conceptuales sobre este fenómeno. Estos artículos pueden clasificarse en dos grupos: mientras algunos autores se han centrado en la influencia que la EO pueda tener en el desempeño de las empresas, otros se han centrado en los factores que pueden influir en la orientación emprendedora de las empresas. En ambos casos hay trabajos donde el constructo EO ha sido medido de dos maneras diferentes: unos siguiendo el modelo de tres dimensiones y otros utilizando el modelo de cinco dimensiones.

En el primer grupo, más extenso que el segundo, el constructo EO es considerado como variable independiente. El objetivo principal de estos trabajos es medir el efecto de EO en el rendimiento de la empresa, cuantificándose esto último a través de diferentes variables como el crecimiento de las ventas e indicadores como el retorno sobre patrimonio (ROE) y el retorno sobre activos (ROA). Algunos de estos artículos son los siguientes: 
Tabla 1

Trabajos donde el constructo EO ha sido tomado como variable independiente y las dimensiones utilizadas.

Artículo Dimensiones de EO

\begin{abstract}
Linking two dimensions of EO to firm performance: the moderating role of environment and industry life cycle. (Lumpkin and Dess, 2001)

Deconstructing the relationship between entrepreneurial orientation and business performance at the embryonic stage of firm growth. (Hughes and Morgan, 2007)

Exploring an inverted u-shape relationship between EO and performance in Chinese ventures (Tang J. et al., 2008)

Entrepreneurial Orientation and Growth of SMEs: a causal model (Moreno and Casillas, 2008)

Entrepreneurial Orientation and firm performance: the role of knowledge creation process (Hui-Li et al. 2009)
\end{abstract}

Entrepreneurial Orientation and New Venture performance: the nodrating role of intra- and extraindustry social capital (Stam and Elrig, 2008)

The effects of EO and Marketing Information on the perfomance of SMEs (Keh et al. 2006)

A configurational approach of the relationship between EO and Growth of FF (Casillas et al. 2010)

Entrepreneurial Orientation and Business Performance- A replication study (Frank et al. 2010)

Entrepreneurial Orientation, Learning Orientation and Firm Perfomance (Wang, 2008)

The moderating impact of internal social exchange processes on the EOperfoamnce relationship (De Clercq et al. 2009)

Contextual influences on the CE-performance relationship: a longitudinal analysis (Zahra and Covin, 1995)

Entrepreneurial Orientation and small business performance: a configurational approach (Wiklund and Sheperd, 2005)

Strategic process effects on the EO-sales growth rate relationship (Covin et al. 2006)

A critical examination of the EO-performance relationship (Andersen, 2010)

Entrepreneurial Orientation, risk taking and performance in family firms (Naldi et al., 2007)
Proactividad, Innovación, Asunción de Riesgos, Agresividad Competitiva

Asunción de Riesgos, Innovación,

Proactividad, Agresividad

Competitiva, Autonomía

Innovación, Proactividad, Asunción de Riesgos

Innovación, Proactividad, Asunción de Riesgos Innovación, Asunción de Riesgos, Proactividad, Agresividad Competitiva, Autonomía

Innovación, Asunción de Riesgos, Proactividad.

Innovación, Asunción de Riesgos, Proactividad.

Innovación, Asunción de Riesgos, Proactividad.

Innovación, Asunción de Riesgos, Proactividad.

Innovación, Asunción de Riesgos, Proactividad, Agresividad Competitiva

Innovación, Asunción de Riesgos, Proactividad.

Innovación, Asunción de Riesgos, Proactividad.

Innovación, Asunción de Riesgos, Proactividad.

Innovación, Asunción de Riesgos, Proactividad.

Innovación, Asunción de Riesgos, Proactividad.

Innovación, Asunción de Riesgos, Proactividad.

Tabla 2

Trabajos donde el constructo EO ha sido tomado como variable dependiente y las dimensiones utilizadas.

\begin{tabular}{|c|c|c|c|c|}
\hline Artículo & \multicolumn{4}{|c|}{ Dimensiones de EO } \\
\hline $\begin{array}{l}\text { Entrepreneurial Orientation of Family Firms: Family and } \\
\text { environmental dimensions (Casillas et al. 2010) }\end{array}$ & $\begin{array}{l}\text { Innovación, } \\
\text { Riesgos }\end{array}$ & Proactividad, & & ción de \\
\hline $\begin{array}{l}\text { EO: the role of institutional environment and firm attributes in } \\
\text { shaping innovation and Proactividad (Dickson, 2004). }\end{array}$ & $\begin{array}{l}\text { Innovación, } \\
\text { Proactividad. }\end{array}$ & Asunción & de & Riesgos, \\
\hline $\begin{array}{l}\text { Corporate Entreprenrurship in Family Firms: a family firms } \\
\text { (Kellermanns and Eddleston, 2006) }\end{array}$ & $\begin{array}{l}\text { Innovación, } \\
\text { Proactividad. }\end{array}$ & Asunción & de & Riesgos, \\
\hline $\begin{array}{l}\text { Entrepreneurial Behavior in Family Firms: a replication study } \\
\text { (Weismeier-Sammer, 2011) }\end{array}$ & $\begin{array}{l}\text { Innovación, } \\
\text { Proactividad. }\end{array}$ & Asunción & de & Riesgos, \\
\hline $\begin{array}{l}\text { Entrepreneurial Orientation in Family Firms: a generational } \\
\text { perspective (Cruz and Nordqvist, 2010) }\end{array}$ & $\begin{array}{l}\text { Innovación, } \\
\text { Proactividad. }\end{array}$ & Asunción & de & Riesgos, \\
\hline $\begin{array}{l}\text { Culture, Entrepreneurial Orientation and Global Competitiveness } \\
\text { (Lee and Peterson, 2011) }\end{array}$ & $\begin{array}{l}\text { Innovación, } \\
\text { Riesgos, } \\
\text { Autonomía }\end{array}$ & $\begin{array}{l}\text { Proactividad, } \\
\text { Agresividad }\end{array}$ & & $\begin{array}{l}\text { ción de } \\
\text { mpetitiva, }\end{array}$ \\
\hline
\end{tabular}


El segundo grupo considera el constructo EO como una variable dependiente. Su principal objetivo es analizar qué factores, tanto internos como externos, tienen influencia en el EO de la empresa familiar y cuantificar dichos efectos. Cabe señalar que son muy abundantes los artículos en donde se analizan los efectos de los factores externos, mientras que los internos no han sido tan estudiados. Algunos de los trabajos más importantes publicados en este sentido son los recogidos en la Tabla 2.

Tal y como se puede observar en la tabla 2, al igual que en el caso donde EO es tomado como una variable independiente, hay un mayor número de estudios que utilizan la escala de medición propuesta por Miller (1983) o la versión modificada propuesta por Covin y Slevin (1989), Covin et al., (1990), y Zahra (1991), donde la innovación, la proactividad y la asunción de riesgos son las dimensiones medidas.

\section{Dimensiones}

Una vez llevada a cabo una revisión bibliográfica sobre la definición y el dominio conceptual de la orientación empresarial, es preciso la realización de un estudio pormenorizado de las cinco dimensiones que componen el constructo EO: la innovación, la proactividad, la asunción de riesgos, la autonomía y la competitividad agresiva.

\subsection{Innovación}

Según Lumpkin y Dess (1996), la innovación, una de las principales dimensiones del EO de una organización es "la propensión de la empresa a participar y apoyar las nuevas ideas, la novedad, la experimentación y los procesos creativos que pueden dar lugar a nuevos productos, servicios, o procesos". Los factores clave de la innovación son la amplitud de miras, la visión compartida y el compromiso con el aprendizaje, de manera que no se limite ni castigue a los empleados de una empresa por cometer errores (Wang, 2008).

Las innovaciones, que reflejan el resultado de una dimensión que ha cobrado importancia en los últimos años debido a los rápidos movimientos de los mercados y a las cambiantes necesidades de los clientes, pueden ser clasificadas según su enfoque, como externas e internas. Las externas representan las innovaciones más visibles en una empresa, que puede tomar la forma de nuevos productos, servicios o procesos tecnológicos. Por el contrario, las internas tienen que ver más con mejoras innovadoras y generadoras de valor a través de renovaciones dentro de las propias empresas (Zellweger y Sieger, 2010).

En el caso específico de las empresas familiares, la innovación, al igual que la autonomía y la proactividad, se considera una de las dimensiones más importantes del EO para la orientación a largo plazo (Nordqvist et al., 2008). Su mayor potencial para alcanzar altos rendimientos suele ir acompañado de una manera integral de tomar decisiones estratégicas (Eddleston et al. 2008) que puede encontrar resistencia en la participación de la familia si es percibida como una amenaza para la identidad de sus familiares (Short et al., 2009).

Zellweger y Sieger (2010) descubrieron que suele haber diferencias entre el nivel de innovación externa e interna. Más concretamente, estos autores indican que, aunque el nivel de los dos tipos de capacidad innovadora fluctúa a largo de la vida de la compañía, las empresas familiares tienden a mantener un nivel entre medio y bajo de innovación externa y entre medio y alto de innovación interna (Zellweger y Sieger, 2010).

Hay algunas razones que pueden explicar este fenómeno. La más extendida aduce que suele haber un alto grado de libertad interna en las empresas mientras que el nivel de libertad externa es menor debido al contexto industrial dominado por las multinacionales. Más aún, los riesgos y los costes de las innovaciones, la lenta aceptación de nuevos productos por parte de los mercados, el uso de tecnologías probadas en entornos estables y el campo de acción elegido por los gerentes-propietarios de empresas familiares hacen más fáciles los cambios internos que los externos (Zellweger et al. 2008). 
Por otro lado, los cambios generacionales en el control de las empresas familiares pueden aumentar tanto el nivel de la innovación interna como el de la externa. No obstante, la generación más reciente en llegar al control tiene que resolver algunos problemas internos sobre la toma de decisiones internas, el tipo de liderazgo y el estilo a seguir antes de considerar los aspectos relacionados con las innovaciones externas (Zellweger y Sieger, 2010).

\subsection{Proactividad}

Otra dimensión que forma el constructo EO es la proactividad. En palabras de Venkataraman (1989), la proactividad se refiere a procesos con el objetivo de prever y operar en las necesidades futuras mediante "la búsqueda de nuevas oportunidades que pueden o no estar relacionados con la línea habitual de actuación, la introducción de nuevos productos y marcas antes que la competencia y la eliminación de las operaciones que estratégicamente están en las etapas de madurez o de declive del ciclo de la vida ".

Puede entenderse como una perspectiva de futuro, basada en el estudio continuo del entorno, donde las empresas prevén oportunidades para desarrollar e introducir nuevos productos con el fin de obtener las ventajas de ser los pioneros y de poder configurar qué dirección toma el propio entorno (Hughes y Morgan, 2007) para de este modo capitalizar las oportunidades emergentes (Wiklund y Shepherd, 2005).

En el caso de las empresas familiares, donde la proactividad se considera la dimensión más importante junto con la autonomía y la innovación (Nordqvist et al., 2008), Zellweger y Sieger (2010) llegaron a dos conclusiones importantes . Una de ellas fue que el patrón del nivel de proactividad en las empresas familiares es dinámico, con algunos períodos de bajos niveles de proactividad $y$ otras fases de movimientos bien estudiados. En ese sentido, Martin y Lumpkin (2003) llegaron a la conclusión de que el nivel de proactividad varía dependiendo de la generación propietaria de las empresas familiares, estando bastante extendida la postura de "esperar y ver" (Zellweger y Sieger, 2010).

Además, Short et al. (2009) indican que en el caso de las empresas familiares de gran tamaño, éstas exhiben menores niveles de proactividad en comparación con las no-familiares. Ésta conclusión está en consonancia con el hecho de que el enfoque estratégico más común empleado por las empresas familiares es la estrategia defensiva. La segunda razón es que la marcada influencia de los miembros familiares que no trabajan en la compañía puede ser una fuente de obstrucción de movimientos proactivos de los CEOs familiares (Zellweger y Sieger, 2010).

\subsection{Asunción de riesgos}

La asunción de riesgos consiste en la realización de acciones audaces como aventurarse en lo desconocido, la contratación masiva y el comprometer gran parte de los recursos en lanzamientos de nuevos productos con un alto grado de incertidumbre (Rauch et al., 2009). En otras palabras, tipifica el grado de voluntad de la gerencia para comprometer recursos de la empresa cuando la decisión tiene una probabilidad considerable de fracaso (Lumpkin y Dess, 1996).

En el caso de las empresas familiares, ha sido ampliamente aceptada la idea de que este tipo de empresas tratan el riesgo de una manera diferente. Esto se debe a que la gestión y la propiedad no están separados y también debido a la naturaleza familiar de la propiedad y la gestión (Carney, 2005; Schulze et al, 2003; Schulze et al, 2001;. Zahra, 2005, Naldi et al 2007).

Así, Naldi et al. (2007) concluyeron que las empresas familiares son más adversas al riesgo debido a que la mayor parte la riqueza de la familia suele estar invertida en la empresa, soportando por completo las hipotéticas pérdidas financieras de las inversiones fallidas. Por otra parte, Nordqvist et al. (2008) llegó a la conclusión de que los niveles de asunción de riesgos y agresividad competitiva son más bajos en las empresas familiares, en comparación con los niveles de proactividad, innovación y autonomía. 


\subsection{Autonomía}

La autonomía puede ser entendida como una acción independiente de un individuo o de un equipo para poner en marcha una idea o una visión y llevarla a cabo hasta su finalización (Lumpkin y Dess, 1996). En otras palabras, se trata de la capacidad y la disposición a decidir libremente por uno mismo para perseguir las oportunidades del mercado.

Dentro del marco conceptual del EO, la autonomía tiene un papel muy importante a la hora de impulsar las fortalezas de una empresa, identificando oportunidades que están fuera de las capacidades de la organización y haciendo de refuerzo para llevar a cabo nuevos proyectos que mejoren las prácticas empresariales (Kanter, et al. 1983). Así, la autonomía refuerza la innovación, estimula la puesta en marcha de proyectos empresariales y mejora la competitividad y la eficacia dentro de las organizaciones (Burgelman, 1984).

Nordqvist et al. (2008) sugieren que la dimensión autonomía aglutina en sí misma dos subdimensiones: una subdimensión externa llamada autonomía externa (autonomía sobre diferentes agentes implicados, tales como bancos, proveedores, clientes y mercados financieros) y una subdimensión interna llamada autonomía interna (autonomía de las personas y equipos dentro de una organización). La autonomía interna es una práctica gerencial más reciente que se da en el lugar de trabajo, siendo incrementado o adoptado, en el caso de las empresas familiares, por las generaciones posteriores siempre que haya presencia de directivos no familiares (Zellweger y Sieger, 2010).

En este tipo de autonomía, las empresas familiares tienden a realizar un menor seguimiento formal y un menor uso de otros mecanismos de control en comparación con las empresas no familiares, siendo ello un requisito importante para la autonomía individual (Eddleston et al., 2008).

Sin embargo, la autonomía externa, entendida como la independencia sobre los agentes externos, ha sido siempre la subdimensión más destacada entre las dos. Este tipo de autonomía puede otorgar a los dueños y administradores la libertad de elegir una estrategia sin tener que satisfacer demandas a corto plazo de de los accionistas, redundando ello en un aumento de la autonomía interna (Zellweger y Sieger, 2010).

A la hora de resumir la relación entre la autonomía y las empresas familiares, Martin y Lumpkin (2003) mostraron que la gestión familiar limita su propia autonomía haciendo partícipes a más personas en la toma de decisiones y la instauración de consejos de administración poderosos, lo que redunda en menores niveles de autonomía que las empresas no familiares (Short et al., 2009).

\subsection{Agresividad competitiva}

La agresividad competitiva se refiere a la tendencia de una empresa de desafiar de manera directa e intensa a sus competidores con el fin de superar a los rivales de su sector (Lumpkin y Dess, 1996).

La agresividad competitiva puede tomar diferentes formas. Algunas de ellas pueden ser la competencia de precios, la entrada al mercado con una nueva o superior oferta, una rápida reacción a las estrategias de los rivales de mercado, la explotación de la información de mercado de forma continua y el uso de tácticas sorpresa o no convencionales. Todas ellas se basan en la adquisición de mayores cuotas de mercado y de clientes atacando las debilidades de los competidores con el fin de atajar su capacidad de competir y también para anticipar y dar respuesta a lo que una empresa agresiva rival espera hacer en el futuro (Hughes y Morgan, 2007).

En el caso de las empresas familiares, sus puntos de vista y sus formas de actuar tienden a ser diferentes a las de las no familiares. De hecho, algunos autores defienden que la agresividad competitiva es, por lo general, menos importante en las empresas familiares (Nordqvist et al., 2008). Dentro de esta dimensión, hay al menos, dos formas diferentes de actuar. Una de ellas es la adopción de una postura agresiva con el fin de deshacer la posición de los competidores, mientras que la 
otra es la actitud conocida como "vive y deja vivir" (Covin y Covin, 1990).

Aunque existen opiniones contrarias sobre la forma en la que actúan las empresas familiares, está más extendida la sensación de su propia necesidad de proyectar una imagen positiva para preservar su identidad puede inhibir las acciones agresivas de carácter ofensivo, ya que estas pueden dañar las percepciones positivas que tengan de ella los diferentes agentes del mercado (Dyer y Whetten, 2006).

Por otra parte, la agresividad competitiva disminuye con el tiempo en las empresas familiares debido al cuidado por parte de las propias familias de su propia reputación (Zellweger y Sieger, 2010).

Así, los gerentes de las empresas familiares se identifican, a menudo, más fuertemente con sus empresas que los gerentes de las compañías no familiares, alcanzando mayores niveles de agresividad solo cuando la empresa se vea amenazada (Gómez-Mejía et al., 2007).

En otras palabras, no van a desarrollar la agresividad competitiva de una manera ofensiva sino de una manera defensiva. Además, hay otro factor que afecta al nivel de intensidad de esta dimensión en las empresas familiares: la participación en el control de la empresa por parte de diferentes generaciones. De hecho, a medida que las generaciones posteriores se van involucrando en el negocio familiar, la agresividad competitiva tiende a ser menor (Martin y Lumpkin, 2003; Zellweger, 2008).

\section{Escalas de medición del constructo Orientación Emprendedora}

De acuerdo a la literatura relacionada con el EO, hay dos maneras de entender este constructo.

Por un lado, algunos autores empezando por Covin y Slevin (1989), perciben el EO como un constructo en la que todas las dimensiones se correlacionan positivamente.

En otras palabras, es necesario alcanzar un alto nivel en todas las dimensiones para tener orientación emprendedora ya que una alta puntuación en una de las dimensiones promoverá un alto nivel en el resto de las mismas. Estos autores utilizan un EO que contiene las siguientes tres dimensiones: la innovación, la proactividad y la asunción de riesgos.

Por otra parte, existe otra parte de la comunidad científica que percibe el EO como un constructo multidimensional que se basa en el trabajo de Lumpkin y Dess (1996).

Este grupo de autores asume que las diferentes dimensiones que componen el EO no necesitan covariar entre sí para catalogar una empresa como emprendedora. Dicho de otro modo, estos autores defienden que una empresa puede obtener altas puntuaciones en algunas de las dimensiones y no en otras, lo cual depende de ciertas variables como el entorno, sin poner en duda la condición emprendedora de una compañía (Lumpkin y Dess, 1996).

En este caso, la idea de que las dimensiones de EO tienden a variar de forma independiente en lugar de covariar está ganando fuerza entre los autores. Estos utilizan un concepto diferente de EO, toda vez que suponen que el constructo EO está compuesto por cinco dimensiones: la innovación, la proactividad, la asunción de riesgos, la autonomía y la agresividad competitiva.

En la tabla 3, hemos recopilado los trabajos empíricos más destacables sobre el EO, donde hemos especificado las dimensiones analizadas, el número de ítems utilizados en total y para cada dimensión y un breve comentario sobre lo más relevante de la escala utilizada.

En dicha tabla se puede observar que, a pesar de que el uso de los dos tipos de escalas está muy extendido, hay más trabajos que han utilizado escalas de tres dimensiones que de cinco.

Los trabajos con estudio empírico realizados mediante una escala de tres dimensiones, se basan principalmente en las escalas diseñadas y desarrolladas por Miller (1983) y Covin y Slevin (1989). 
Dimensiones, ítems y escalas de medición de estudios empíricos en artículos sobre el constructo EO.

\begin{tabular}{|c|c|c|c|c|}
\hline Artículo & Dimensiones & $\begin{array}{c}\text { Num. } \\
\text { Ítems } \\
" n\end{array}$ & Ítems & Escala de medición \\
\hline $\begin{array}{l}\text { Strategic management of small firms in } \\
\text { hostile and benign environments (Covin } \\
\text { and Slevin, 1989) }\end{array}$ & $\begin{array}{l}\text { Innovación, } \\
\text { Proactividad, } \\
\text { Asunción de } \\
\text { Riesgos }\end{array}$ & 9 & $\begin{array}{l}3 \text { para Innovación; } \\
3 \text { para } \\
\text { Proactividad; } 3 \\
\text { para Asunción de } \\
\text { Riesgos. }\end{array}$ & $\begin{array}{l}\text { 1976). La escala más utilizada. Su } \\
\text { origen radica en la escala de } \\
\text { Khandawalla (1976). }\end{array}$ \\
\hline $\begin{array}{l}\text { Clarifying the Entrepreneurial Orientation } \\
\text { Construct and linking it to Performance } \\
\text { (Lumpkin and Dess, 1996) }\end{array}$ & $\begin{array}{l}\text { Asunción de } \\
\text { Riesgos, } \\
\text { Innovación, } \\
\text { Proactividad, } \\
\text { Agresividad } \\
\text { Competitiva, } \\
\text { Autonomía } \\
\end{array}$ & N/A & N/A & $\begin{array}{l}\text { Artículo seminal de conceptualización } \\
\text { de las } 5 \text { dimensiones de EO. }\end{array}$ \\
\hline $\begin{array}{l}\text { Linking two dimensions of EO to firm } \\
\text { performance: the moderating role of } \\
\text { environment and industry life cycle. } \\
\text { (Lumpkin and Dess, 2001) }\end{array}$ & $\begin{array}{l}\text { Proactividad, } \\
\text { Innovación, } \\
\text { Asunción de } \\
\text { Riesgos, } \\
\text { Agresividad } \\
\text { Competitiva }\end{array}$ & 11 & $\begin{array}{l}3 \text { para } \\
\text { Proactividad; } 3 \\
\text { para Innovación; } 3 \\
\text { para Asunción de } \\
\text { Riesgos; 2 para } \\
\text { Agresividad } \\
\text { Competitiva. }\end{array}$ & $\begin{array}{l}2 \text { para Proactividad, } 1 \text { basado en Covin } \\
\text { y Slevin (1989) y } 1 \text { de contribución } \\
\text { propia. } 3 \text { para Innovación de Covin y } \\
\text { Slevin (1989); } 3 \text { para Asunción de } \\
\text { Riesgos de Covin y Slevin (1989); } 2 \\
\text { para Agresividad Competitiva de } \\
\text { contribución propia. }\end{array}$ \\
\hline $\begin{array}{l}\text { Understanding and measuring autonomy: } \\
\text { an EO perspective (Lumpkin et al., 2009) }\end{array}$ & $\begin{array}{l}\text { Proactividad, } \\
\text { Asunción de } \\
\text { Riesgos, } \\
\text { Agresividad } \\
\text { Competitiva } \\
\qquad 22 \\
\end{array}$ & 22 & $\begin{array}{l}\text { Autonomía, } \\
\text { Innovación }\end{array}$ & $\begin{array}{l}8 \text { para Autonomía de contribución } \\
\text { propia; } 3 \text { para Innovación basado en } \\
\text { Covin y Slevin (1989) y } 2 \text { de Lumpkin } \\
\text { (1998); } 3 \text { para Proactividad basado en } \\
\text { Covin y Slevin (1989) y } 1 \text { de Lumpkin } \\
\text { y Dess (2001); } 3 \text { para Asunción }\end{array}$ \\
\hline $\begin{array}{l}\text { Cross-cultural reliability and validity of a } \\
\text { scale to measure firm entrepreneurial } \\
\text { orientation (Knight, 1997). }\end{array}$ & $\begin{array}{l}\text { Innovación, } \\
\text { Proactividad, } \\
\text { Asunción de } \\
\text { Riesgos }\end{array}$ & 8 & $\begin{array}{l}3 \text { para Innovación; } \\
2 \text { para } \\
\text { Proactividad; } 3 \\
\text { para Asunción de } \\
\text { Riesgos. }\end{array}$ & $\begin{array}{l}3 \text { para Innovación basado en Covin y } \\
\text { Slevin (1989); } 2 \text { para Proactividad } \\
\text { basado en Covin y Slevin (1989); } 3 \\
\text { para Asunción de Riesgos basado en } \\
\text { Covin y Slevin (1989). }\end{array}$ \\
\hline $\begin{array}{l}\text { Internal capabilities, external networks, } \\
\text { and performance: A study based } \\
\text { on technology based ventures.(Lee, C. et } \\
\text { al. 2001). }\end{array}$ & $\begin{array}{l}\text { Innovación, } \\
\text { Asunción de } \\
\text { Riesgos, } \\
\text { Proactividad }\end{array}$ & 6 & $\begin{array}{l}2 \text { para Innovación; } \\
2 \text { para Asunción } \\
\text { de Riesgos; } 2 \text { para } \\
\text { Proactividad; }\end{array}$ & $\begin{array}{l}2 \text { para Innovación; } 2 \text { para Asunción de } \\
\text { Riesgos; } 2 \text { para Proactividad; } 2 \text { para } \\
\text { Innovación, de contribución propia; } 2 \\
\text { para Asunción de Riesgos,de } \\
\text { contribución propia; } 2 \text { para Proactividad } \\
\text { de contribución propia. No utiliza la } \\
\text { escala Likert, utiliza una escala } \\
\text { numérica. }\end{array}$ \\
\hline $\begin{array}{l}\text { Internal capabilities, external networks, } \\
\text { and performance: A study based } \\
\text { on technology based ventures.(Lee, C. et } \\
\text { al. 2001). Innovación, Asunción de } \\
\text { Riesgos, Proactividad }\end{array}$ & $\begin{array}{l}\text { Innovación, } \\
\text { Asunción de } \\
\text { Riesgos, } \\
\text { Proactividad }\end{array}$ & 6 & $\begin{array}{l}2 \text { para Innovación; } \\
2 \text { para Asunción } \\
\text { de Riesgos; } 2 \text { para } \\
\text { Proactividad; }\end{array}$ & $\begin{array}{l}2 \text { para Innovación, de contribución } \\
\text { propia; } 2 \text { para Asunción de Riesgos,de } \\
\text { contribución propia; } 2 \text { para Proactividad } \\
\text { de contribución propia. No utiliza la } \\
\text { escala Likert, utiliza una escala } \\
\text { numérica. }\end{array}$ \\
\hline $\begin{array}{l}\text { Deconstructing the relationship between } \\
\text { entrepreneurial orientation and business } \\
\text { performance at the embryonic stage of } \\
\text { firm growth. (Hughes and Morgan, 2007) }\end{array}$ & $\begin{array}{l}\text { Asunción de } \\
\text { Riesgos, } \\
\text { Innovación, } \\
\text { Proactividad, } \\
\text { Agresividad } \\
\text { Competitiva, } \\
\text { Autonomía } \\
\end{array}$ & 18 & $\begin{array}{l}3 \text { para Asunción } \\
\text { de Riesgos; } 3 \text { para } \\
\text { Innovación; } 3 \text { para } \\
\text { Proactividad; } 3 \\
\text { para Agresividad } \\
\text { Competitiva:; } 6 \\
\text { para Autonomía; }\end{array}$ & $\begin{array}{l}\text { Todos los item son de contribución } \\
\text { propia }\end{array}$ \\
\hline $\begin{array}{l}\text { Exploring an inverted u-shape } \\
\text { relationship between EO and performance } \\
\text { in Chinese ventures (Tang J. et al., 2008) }\end{array}$ & $\begin{array}{l}\text { Innovación, } \\
\text { Proactividadt, } \\
\text { Asunción de } \\
\text { Riesgos }\end{array}$ & 8 & $\begin{array}{l}3 \text { para Innovación; } \\
3 \text { para } \\
\text { Proactividad; } 2 \\
\text { para Asunción de } \\
\text { Riesgos; }\end{array}$ & $\begin{array}{l}\text { Covin y Slevin (1989) pero sin uno de } \\
\text { los item para Asunción de Riesgos. }\end{array}$ \\
\hline $\begin{array}{l}\text { Cross-national invariance of the EO scale } \\
\text { (Hansen et al., 2011) }\end{array}$ & $\begin{array}{l}\text { Innovación, } \\
\text { Proactividadt, } \\
\text { Asunción de } \\
\text { Riesgos }\end{array}$ & 8 & $\begin{array}{l}3 \text { para Innovación; } \\
3 \text { para } \\
\text { Proactividad;2 } \\
\text { para Asunción de } \\
\text { Riesgos; }\end{array}$ & $\begin{array}{l}\text { Covin y Slevin (1989) pero sin uno de } \\
\text { los item para Asunción de Riesgos. }\end{array}$ \\
\hline $\begin{array}{l}\text { Entrepreneurial Orientation and Growth } \\
\text { of SMEs: a causal model (Moreno and } \\
\text { Casillas, 2008) }\end{array}$ & $\begin{array}{l}\text { Innovación, } \\
\text { Proactividadt, } \\
\text { Asunción de } \\
\text { Riesgos }\end{array}$ & 13 & $\begin{array}{lr}5 & \text { para } \\
\text { Innovación,4 } & \text { para } \\
\text { Asunción } & \text { de } \\
\text { Riesgos, } 4 & \text { para } \\
\text { Proactividad. } & \end{array}$ & $\begin{array}{l}5 \text { para Innovación: } 3 \text { para Lumpkin } \\
\text { (1998) y } 2 \text { de contribución propia; } \\
4 \text { para Asunción de Riesgos: } 3 \text { de } \\
\text { Lumpkin (1998) y } 1 \text { de contribución } \\
\text { propia y } 4 \text { para Proactividad: } 3 \text { de } \\
\text { Lumpkin (1998) y } 1 \text { de contribución } \\
\text { propia." }\end{array}$ \\
\hline
\end{tabular}




\begin{tabular}{|c|c|c|c|c|}
\hline Artículo & Dimensiones & $\begin{array}{l}\text { Num. } \\
\text { Ítems } \\
"\end{array}$ & Ítems & Escala de medición \\
\hline $\begin{array}{l}\text { EO in cross cultural research: assessing } \\
\text { measurement invariance in the Construct } \\
\text { (Runyan and Ge, 2011) }\end{array}$ & $\begin{array}{l}\text { Innovación, } \\
\text { Proactividad, } \\
\text { Asunción de } \\
\text { Riesgos }\end{array}$ & 8 & $\begin{array}{l}3 \text { para Innovación; } \\
3 \text { para } \\
\text { Proactividad; } 2 \\
\text { para Asunción de } \\
\text { Riesgos. }\end{array}$ & $\begin{array}{l}\text { Covin y Slevin (1989) pero sin uno de } \\
\text { los item para Asunción de Riesgos. }\end{array}$ \\
\hline $\begin{array}{l}\text { Entrepreneurial Orientation of Family } \\
\text { Firms: Family and environmental } \\
\text { dimensions (Casillas et al. 2010) }\end{array}$ & $\begin{array}{l}\text { Innovación, } \\
\text { Proactividad, } \\
\text { Asunción de } \\
\text { Riesgos }\end{array}$ & 9 & $\begin{array}{l}3 \text { para Innovación; } \\
3 \text { para } \\
\text { Proactividad; } 3 \\
\text { para Asunción de } \\
\text { Riesgos }\end{array}$ & Covin y Slevin (1989) \\
\hline $\begin{array}{l}\text { EO: the role of institutional environment } \\
\text { and firm attributes in shaping innovation } \\
\text { and Proactividad (Dickson, 2004). }\end{array}$ & $\begin{array}{l}\text { Innovación, } \\
\text { Asunción de } \\
\text { Riesgos, } \\
\text { Proactividad. }\end{array}$ & 5 & $\begin{array}{l}3 \text { para Innovación; } \\
3 \text { para } \\
\text { Proactividad; } 2 \\
\text { para Asunción de } \\
\text { Riesgos }\end{array}$ & Basado en Covin y Slevin (1989) \\
\hline $\begin{array}{l}\text { Entrepreneurial Orientation and Mew } \\
\text { Venture performance: the nodrating role of } \\
\text { intra- and extraindustry social capital (Stam } \\
\text { and Elrig, 2008) }\end{array}$ & $\begin{array}{l}\text { Innovación, } \\
\text { Asunción de } \\
\text { Riesgos, } \\
\text { Proactividad. }\end{array}$ & 9 & $\begin{array}{l}3 \text { para Innovación; } \\
3 \text { para } \\
\text { Proactividad; } 3 \\
\text { para Asunción de } \\
\text { Riesgos } \\
\end{array}$ & $\begin{array}{l}\text { Basado en Covin y Slevin (1989), y } \\
\text { reemplazado uno por otro del de } \\
\text { Lumpkin y Dess (2001) }\end{array}$ \\
\hline $\begin{array}{l}\text { The effects of EO andMarketing } \\
\text { Information on the perfomance of SMEs } \\
\text { (Keh et al. 2006) }\end{array}$ & $\begin{array}{l}\text { Innovación, } \\
\text { Asunción de } \\
\text { Riesgos, } \\
\text { Proactividad. }\end{array}$ & 8 & $\begin{array}{l}3 \text { para Innovación, } \\
2 \text { para Asunción } \\
\text { de Riesgos , } 3 \\
\text { para Proactividad: }\end{array}$ & $\begin{array}{l}\text { Basado en Covin y Slevin (1989) y } \\
\text { Miller y Friesen (1982) }\end{array}$ \\
\hline $\begin{array}{l}\text { A configurational approach of the } \\
\text { relationship between EO and Growth of FF } \\
\text { (Casillas et al. 2010) }\end{array}$ & $\begin{array}{l}\text { Innovación, } \\
\text { Asunción de } \\
\text { Riesgos, } \\
\text { Proactividad } \\
\end{array}$ & 9 & $\begin{array}{l}4 \text { para Innovación, } \\
3 \text { para Asunción } \\
\text { de Riesgos , } 2 \\
\text { para Proactividad: }\end{array}$ & $\begin{array}{l}\text { Utilizado por Lumpkin (1998) y } \\
\text { Lumpkin y Dess (2001) }\end{array}$ \\
\hline $\begin{array}{l}\text { Entrepreneurial Orientation and Business } \\
\text { Performance- A replication study (Frank et } \\
\text { al. 2010) }\end{array}$ & $\begin{array}{l}\text { Innovación, } \\
\text { Asunción de } \\
\text { Riesgos, } \\
\text { Proactividad }\end{array}$ & 8 & $\begin{array}{l}3 \text { para Innovación } \\
, 2 \text { para Asunción } \\
\text { de Riesgos, } 3 \text { para } \\
\text { Proactividad }\end{array}$ & $\begin{array}{l}\text { Basado en Miller (1983; 1987a) y } \\
\text { utilizado por Wiklund y Sheperd (2005) }\end{array}$ \\
\hline $\begin{array}{l}\text { Entrepreneurial Orientation, Learning } \\
\text { Orientation and Firm Perfomance (Wang, } \\
\text { 2008) }\end{array}$ & $\begin{array}{l}\text { Innovación, } \\
\text { Asunción de } \\
\text { Riesgos, } \\
\text { Proactividad, } \\
\text { Agresividad } \\
\text { Competitiva }\end{array}$ & 11 & $\begin{array}{l}3 \text { para Innovación, } \\
3 \text { para } \\
\text { Proactividad, } 3 \\
\text { para Asunción de } \\
\text { Riesgos y } 2 \text {.para } \\
\text { Competitve } \\
\text { Aggressiveness. }\end{array}$ & $\begin{array}{l}\text { Basado en Miller (1983) y Covin y } \\
\text { Slevin (1989). La Agresividad } \\
\text { Competitiva está medida según } \\
\text { Lumpkin y Dess (1996) }\end{array}$ \\
\hline $\begin{array}{l}\text { The moderating impact of internal social } \\
\text { exchange processes on the EO-perfoamnce } \\
\text { relationship (De Clercq et al. 2009) }\end{array}$ & $\begin{array}{l}\text { Innovación, } \\
\text { Asunción de } \\
\text { Riesgos, } \\
\text { Proactividad }\end{array}$ & 7 & $\begin{array}{l}2 \text { para Innovación, } \\
3 \text { para } \\
\text { Proactividad y } 3 \\
\text { para Asunción de } \\
\text { Riesgos } \\
\end{array}$ & $\begin{array}{l}\text { Basado en Covin y Slevin (1989) y } \\
\text { Miller (1983) }\end{array}$ \\
\hline $\begin{array}{l}\text { Entrepreneurial Orientation and small } \\
\text { business performance: a configurational } \\
\text { approach (Wiklund and Sheperd, 2005) }\end{array}$ & $\begin{array}{l}\text { Innovación, } \\
\text { Asunción de } \\
\text { Riesgos, } \\
\text { Proactividad }\end{array}$ & 8 & $\begin{array}{l}3 \text { para Innovación, } \\
3 \text { para } \\
\text { Proactividad y } 2 \\
\text { para Asunción de } \\
\text { Riesgos. }\end{array}$ & $\begin{array}{l}\text { Basado en Covin y Slevin (1989) y } \\
\text { Miller (1983) }\end{array}$ \\
\hline $\begin{array}{l}\text { Corporate Entrepreneurship in Family } \\
\text { Firms: a family firms (Kellermanns and } \\
\text { Eddleston, 2006) }\end{array}$ & $\begin{array}{l}\text { Innovación, } \\
\text { Asunción de } \\
\text { Riesgos, } \\
\text { Proactividad. }\end{array}$ & 7 & $\begin{array}{l}3 \text { para Innovación, } \\
2 \text { para } \\
\text { Proactividad y } 2 \\
\text { para Asunción de } \\
\text { Riesgos. }\end{array}$ & $\begin{array}{l}\text { Basado en Covin y Slevin (1989) y } \\
\text { Miller (1983) }\end{array}$ \\
\hline $\begin{array}{l}\text { Entrepreneurial Behavior in Family Firms: a } \\
\text { replication study (Weismeier-Sammer, } \\
\text { 2011) }\end{array}$ & $\begin{array}{l}\text { Innovación, } \\
\text { Asunción de } \\
\text { Riesgos, } \\
\text { Proactividad }\end{array}$ & 7 & $\begin{array}{l}3 \text { para Innovación, } \\
2 \text { para } \\
\text { Proactividad y } 2 \\
\text { para Asunción de } \\
\text { Riesgos. }\end{array}$ & $\begin{array}{l}\text { Basado en Covin y Slevin (1989) y } \\
\text { Miller (1983) }\end{array}$ \\
\hline $\begin{array}{l}\text { Entrepreneurial Orientation in family firms: } \\
\text { a generational perspective (Cruz and } \\
\text { Nordqvist, 2010) }\end{array}$ & $\begin{array}{l}\text { Innovación, } \\
\text { Asunción de } \\
\text { Riesgos, } \\
\text { Proactividad }\end{array}$ & 9 & $\begin{array}{l}3 \text { para Innovación, } \\
3 \text { para } \\
\text { Proactividad y } 3 \\
\text { para Asunción de } \\
\text { Riesgos. }\end{array}$ & $\begin{array}{l}\text { Basado en Covin y Slevin (1989) y } \\
\text { Miller (1983) }\end{array}$ \\
\hline
\end{tabular}




\begin{tabular}{|c|c|c|c|c|}
\hline Artículo & Dimensiones & $\begin{array}{c}\text { Num. } \\
\text { Ítems } \\
"\end{array}$ & Ítems & Escala de medición \\
\hline $\begin{array}{l}\text { An investigation of EO, perceived } \\
\text { environmental hostility, and strategy } \\
\text { application among chinese SMEs (Tang and } \\
\text { Hull, 2012) }\end{array}$ & $\begin{array}{l}\text { Innovación, } \\
\text { Asunción de } \\
\text { Riesgos, } \\
\text { Proactividad. }\end{array}$ & 8 & $\begin{array}{l}3 \text { para Innovación, } \\
3 \text { para } \\
\text { Proactividad y } 2 \\
\text { para Asunción de } \\
\text { Riesgos }\end{array}$ & $\begin{array}{l}\text { Basado en Covin y Slevin (1989) y } \\
\text { Miller (1983) }\end{array}$ \\
\hline $\begin{array}{l}\text { Strategic process effects on the EO-sales } \\
\text { growth rate relationship (Covin et al. 2006) }\end{array}$ & $\begin{array}{l}\text { Innovación, } \\
\text { Asunción de } \\
\text { Riesgos, } \\
\text { Proactividad. }\end{array}$ & 7 & Not specified & $\begin{array}{l}\text { Basado en Covin y Slevin (1989) y } \\
\text { Miller (1983) }\end{array}$ \\
\hline $\begin{array}{l}\text { Entrepreneurial Orientation, risk taking and } \\
\text { performance in family firms (Naldi et al., } \\
2007 \text { ) }\end{array}$ & $\begin{array}{l}\text { Asunción de } \\
\text { Riesgos, } \\
\text { Innovación, } \\
\text { Proactividad }\end{array}$ & 9 & $\begin{array}{l}3 \text { para Innovación, } \\
3 \text { para } \\
\text { Proactividad y } 3 \\
\text { para Asunción de } \\
\text { Riesgos. }\end{array}$ & Adaptado de Covin y Slevin (1989) \\
\hline
\end{tabular}

Estas escalas están, en su mayoría, compuestas por entre siete y nueve ítems, donde tres de ellos se utilizan para medir la innovación, dos o tres para medir la proactividad y el resto para la asunción de riesgos.

Por su parte, los artículos con una escala de cinco dimensiones tienen más ítems y se basan principalmente en el trabajo de Lumpkin y Dess (1996), aunque hay más contribuciones propias por parte de algunos autores sobre las dimensiones de autonomía y de agresividad competitiva. En la literatura referente a las empresas familiares, no hay una tendencia clara acerca del uso de la escala de tres dimensiones o la utilización de la escala basada en cinco dimensiones. Las características específicas de este tipo de empresas pueden tener efectos, especialmente, en las dimensiones de autonomía y de agresividad competitiva.

En el caso de la autonomía, las subdimensiones interna y externa de la misma puede verse afectadas por la especificidad de las empresas familiares (Nordqvist et al, 2008; Zellweger y Sieger, 2010). Así, con respecto a la autonomía interna, este tipo de empresas tienden a utilizar menos la supervisión formal y los mecanismos de control en comparación con las empresas no familiares, siendo ello un requisito importante para la autonomía individual (Eddleston et al., 2008). Por lo tanto, las empresas familiares pueden tener menores valores de autonomía interna que las empresas no familiares. Por otro lado, las empresas familiares suelen tener un menor nivel de endeudamiento que las empresas no familiares debido al conservadurismo de la que suelen hacer gala. Así, la autonomía externa tiende a ser más alta que en las empresas no familiares, impulsando de esta manera la autonomía interna a niveles más altos (Zelweger y Sieger, 2010). Por todo ello, se hace necesario medir la dimensión de autonomía para la cuantificación de estos dos efectos antagónicos.

En lo que a la dimensión de agresividad competitiva hace referencia, las empresas familiares tienden a evitar las conductas agresivas de carácter muy ofensivo para no dañar la imagen de la empresa. Por esa razón, suelen elegir la postura estratégica de "vive y deja vivir" (Covin y Covin, 1990) y sólo suele actuar con agresividad competitiva en los casos en que la empresa realmente se siente amenazada, desarrollando en este caso una postura defensiva de agresividad competitiva. Esta estrategia contrasta con los altos niveles de agresividad competitiva ofensiva que las empresas no familiares tienden a tener. Por otro lado, también puede tener efectos sobre la agresividad competitiva de una empresa familiar qué generación ostenta el control de la compañía. De hecho, cuando las generaciones posteriores asumen el control de la misma, la agresividad competitiva tiende a ser menor (Martin y Lumpkin, 2003; Zellweger, 2008). Por ello, resulta más que interesante medir la dimensión agresividad competitiva en las empresas familiares.

Por todo lo expuesto anteriormente, estimamos más conveniente la utilización de una escala de cinco dimensiones en lugar de una de tres dimensiones, midiendo así las dimensiones de autonomía y de agresividad competitiva. 
No obstante, en la tabla 3 se puede observar que, aunque la utilización de ambos tipos de escala está muy extendida, hay más escalas de tres dimensiones que de cinco. Los artículos con estudios empíricos realizados utilizando una escala de tres dimensiones, son los basados principalmente en las escalas diseñadas $\mathrm{y}$ desarrolladas por Miller (1983) y Covin y Slevin (1989). Estas escalas están, la mayoría de veces, compuestas por entre siete y nueve ítems, donde tres de ellos se utilizan para medir la innovación, dos o tres para medir la proactividad y el resto para la medición de la asunción de riesgo.

Por otra parte, los artículos con una escala de cinco dimensiones de EO tienen más ítems y están basados principalmente en el trabajo de Lumpkin y Dess (1996), aunque hay más contribuciones propias de algunos autores sobre la medición de las dimensiones de autonomía y de agresividad competitiva.

\section{Comentarios generales}

En este trabajo, después de realizar una profunda revisión del constructo EO y de sus dimensiones en el que nos hemos centrado en el caso específico de las empresas familiares, hemos analizado las dos principales formas de entender éste constructo. Así, en relación con estas dos percepciones existen dos tendencias distintas a la hora de medir el constructo EO. Algunos autores utilizan escalas de 3 dimensiones, mientras que otros prefieren escalas que albergan 5 dimensiones. En este punto llama la atención lo extendido que están las escalas de 3 dimensiones, ya que no miden algunas de las características de las dimensiones de autonomía y agresividad competitiva. Además, los modelos de 3 dimensiones se basan en la necesidad de que todas las dimensiones deben de tener un peso relativo considerable, teniendo que covariar unas dimensiones con otras. Estas premisas chocan con los distintos tipos de empresas de hoy en día, muchas de los cuales pertenecen a sectores totalmente diferentes. Más aún, en estos modelos no se consideran las características derivadas de las diferentes etapas del ciclo de negocio de una empresa. Estas variables nos pueden llevar a pensar que todas las empresas pueden no tener un peso relativo considerable en todas las dimensiones, ni la necesidad de covariar entre ellos. Así, las empresas se centrarán más en algunas dimensiones que en otras, sin perder por ello la orientación emprendedora de su gestión.

Sin embargo, el hecho de que las escalas de 5 dimensiones no estén tan extendidas puede deberse, en gran medida, a la falta de escalas de las dimensiones de autonomía y agresividad competitiva que estén totalmente establecidas y comúnmente aceptadas. Prueba de ello es el gran número de contribuciones en los últimos años realizadas por diferentes autores que no han alcanzado el apoyo de la comunidad científica. Por ello, creemos que existe la necesidad de un desarrollo mayor de las escalas de autonomía y agresividad competitiva, con el fin de que puedan ser, posteriormente, establecidas y aceptadas por la comunidad científica.

En el caso de las empresas familiares, donde algunas de sus características específicas pueden provocar diferencias con respecto a las no familiares, resulta más conveniente la utilización de una escala donde se midan las dimensiones de autonomía y agresividad competitiva. Tal y como se afirmaba anteriormente, las características específicas de las empresas familiares pueden provocar un doble efecto en la dimensión de autonomía. Por un lado, este tipo de empresas suelen establecer unos controles menos formales que las empresas no familiares y tienden a poner en práctica menos mecanismos de control, lo que hace bajar el nivel de autonomía interna (Eddleston et al., 2008). Por otro lado, el conservadurismo de las empresas familiares hace que tengan unos niveles menores de endeudamiento que las empresas no familiares, por lo que su autonomía externa tiende a ser mayor, impulsando de esta manera la autonomía interna a niveles superiores. Por todo ello, resulta conveniente medir la dimensión de autonomía. En lo que respecta a la dimensión de agresividad competitiva, variables como qué generación familiar tiene el control de la firma y otras características inherentes a este tipo de 
empresa, como puede ser la voluntad de evitar los comportamientos agresivos con el fin de no dañar la imagen de la compañía, hacen necesario medir esta dimensión en las empresas de carácter familiar.

De esta manera, los estudios del constructo Orientación Emprendedora en las empresas familiares tendrán en cuenta dos aspectos que pueden ser la causa de algunas de las principales diferencias entre las empresas familiares y no familiares. Por todo ello, consideramos más apropiado el uso de una escala de cinco dimensiones para medir el constructo EO en el caso de las empresas familiares.

\section{Referencias}

Andersen, J. (2010). A critical examination of the EO-performance relationship. Entrepreneurial behavior and Research, 16(4), 309-328.

Astrachan, J. H. (2003). Commentary on the special issue: The emergence of a field. Journal of Business Venturing, 18, 567-572.

Baird, I.S. and Thomas, H. (1985). Toward a contingency model of strategic risk taking. Academy of Management Review, 10, 230-243.

Barney, J. (1991). Firm resources and sustained competitive advantage. Journal of Management, 17, 99-119.

Birkinshaw, J. (1997) "Entrepreneurship in multinational corporations: the characteristics of subsidiary initiatives". Strategic Management Journal, 18(3):207-229

Burgelman, R. A. (1984). Designs for corporate entrepreneurship in established firms. California Management Review, 26(3), 154-166.

Carney, M. (2005). Corporate governance and competitive advantage in family-controlled firms. Entrepreneurship, Theory and Practice, 29(3), 249265

Casillas, J. C., Moreno, A. M., \& Barbero, J. L. (2010). A configurational approach of the relationship between entrepreneurial orientation and growth of family firms. Family Business Review, 23(1), 27.

Casillas, J. C., Moreno, A. M., \& Barbero, J. L. (2011). Entrepreneurial orientation of family firms: Family and environmental dimensions. Journal of Family Business Strategy, 2 (2), 90-100.

Covin J.G. and Covin, T. (1990). Competitive aggressiveness, environmental context, and small firm performance. Entrepreneurship: Theory and Practice, 14(4), 35-50.

Covin, J.G., Green, K.M., and Slevin, D.P. (2006). Strategic process effects on the entrepreneurial orientation sales growth rate relationship. Entrepreneurship Theory and Practice, 30(1), 57-81.

Covin, J.G. and Miles, M.P. (1999). Corporate entrepreneurship and the pursuit of competitive advantage.Entrepreneurship Theory and Practice, 23(3), 47-63.

Covin, J. G., and Slevin, D. P. (1989). Strategic management of small firms in hostile and benign environments. Strategic Management Journal, 10, 75-87.

Covin J.G. and Wales, W.J. (2012). The measurement of Entrepreneurial Orientation. Entrepreneurship: Theory and Practice, 14(4), 3550. Entrepreneurship Theory and Practice, 36(4), 677-702.

Cruz, C. and Nordqvist, M. (2010). Entrepreneurial orientation in family firms: a generational perspective. Small Business Economics, DOI 10.1007/s11187-010-9265-8.

De Clercq, D., Dimov, D., \& Thongpapanl, N. T. (2010). The moderating impact of internal social exchange processes on the entrepreneurial orientation-performance relationship. Journal of Business Venturing, 25(1), 87-103.

Dickson, P. H. (2004). Entrepreneurial orientation: The role of institutional environment and firm attributes in shaping innovation and proactiveness. Pat, 404, 894-4372.

Dyer, G., and Whetten, D. A. (2006). Family firms and social responsibility: Preliminary evidence from the SandP 500. Entrepreneurship Theory and Practice, 30(6), 785-802.

Eddleston, K., Kellermanns, F. W., and Zellweger, T. (2008). Corporate entrepreneurship in family firms: A stewardship perspective. Paper presented at the USASBE, San Antonio.

Frank, H.; Kessler, A. and Fink, M. (2010) Entrepreneurial Orientation and Business Performance- A replication study. Schmalenbach Business Review, 62, 175-198.

George, B. A., \& Marino, L. (2011). The epistemology of entrepreneurial orientation: Conceptual formation, modeling, and operationalization. Entrepreneurship Theory and Practice, 35 (5). 989-1024.

Gómez-Mejía, L., Haynes, K., Núñez-Nickel, M., Jacobson, K., and Moyano-Fuentes, J., (2007), Socioemotional wealth and business risks in familycontrolled firms: Evidence from Spanish olive oil 
mills, Administrative Science Quarterly, 52(1), 106137.

Guth, W.; Ginsberg, A (1990) Guest Editor's Introduction: "Corporate Entrepreneurship". Strategic Management Journal, 11:5-15

Hansen, J.D.; Deitz, G.D.; Tokman, M.; Marino, L.D.; Weaver, K.M. (2011) Cross-national invariance of the EO scale. Journal of Business Venturing, 26 (1), 61-78.

Hughes, M. and Morgan, R.E. (2007). Deconstructing the relationship between entrepreneurial orientation and business performance at the embryonic stage of firm growth. Industrial Marketing Management, 36, 651-661.

Hui Li,Y.; Wen Huang, J., Tien Tsai, M. (2009) Entrepreneurial Orientation and firm performance: the role of knowledge creation process. Industrial Marketing Management, 38 (4), 440-449.

Ireland, R. D., Hitt, M. A., \& Sirmon, D. G. (2003). A model of strategic entrepreneurship: The construct and its dimensions. Journal of Management, 29(6), 963-989.

Jantunen, A., Puumalainen, K., Saarenketo, S., and Kyläheiko, K. (2005). Entrepreneurialorientation, dynamic capabilities and international performance. Journal of International Entrepreneurship, 3(3), 223.

Kanter, R. M. (1982). The middle manager as innovator. Harvard Business Review, 60(4), 95-106.

Kanter, R. M. (1983). The change masters: Innovation and entrepreneurship in the American corporation, New York: Simon and Schuster.

Keh, H. T., Nguyen, T. T. M., \& Ng, H. P. (2007). The effects of entrepreneurial orientation and marketing information on the performance of SMEs. Journal of Business Venturing, 22(4), 592611.

Kellermanns, F. W., and Eddleston, K. A. (2006). Corporate entrepreneurship in family firms: A family perspective. Entrepreneurship Theory and Practice, 30, 809-830.

Knight, G. A. (1997). Cross-cultural reliability and validity of a scale to measure firm entrepreneurial orientation. Journal of Business Venturing, 12(3), 213-225.

Kuratko, D. F. (2010). Corporate entrepreneurship: An introduction and research review. Handbook of Entrepreneurship Research, 129-163.

Kuratko, D. F., Ireland, R. D., \& Hornsby, J. S. (2004). Corporate entrepreneurship behavior among managers: A review of theory research and practice. Advances in Entrepreneurship, Firm Emergence and Growth, 7, 7-45.
Lee, C., Lee, K., \& Pennings, J. M. (2001). Internal capabilities, external networks, and performance: A study on technology-based ventures. Strategic Management Journal, 22(6-7), 615-640.

Lee, S. M., Lim, S., \& Pathak, R. D. (2011). Culture and entrepreneurial orientation: A multicountry study. International Entrepreneurship and Management Journal, 7(1), 1-15.

Lumpkin, G., Cogliser, C., and Schneider, D. (2009). Understanding and measuring autonomy: An entrepreneurial orientation perspective. Entrepreneurship Theory and Practice, 33(1), 47.

Lumpkin, G. T., and Dess, G. G. (1996). Clarifying the entrepreneurial orientation construct and linking it to performance. Academy of Management Review, 21(1), 135-172.

Lumpkin, G.T. and Dess, G.G. (2001). Linking two dimensions of entrepreneurial orientation to firm performance: The moderating role of environment and industry life cycle. Journal of Business Venturing, 16(5), 429-451.

Martin, W., and Lumpkin, G. T. (2003). From entrepreneurial orientation to family orientation: generational differences in the management of family businesses. Paper presented at the Babson College Entrepreneurship Research Conference, Babson College, Wellesley, MA, USA.

Miller, D. (1983). The correlates of entrepreneurship in three types of firms. Management Science, 29, 770-792.

Moreno, A. M. and Casillas, J.C., (2008) Entrepreneurial Orientation and Growth of SMEs: a causal model. Entrepreneurship Theory and Practice, 32 (2), 507-528.

Morris, M. H., Kuratko, D. F., \& Covin, J. G. (2010). Corporate entrepreneurship \& innovation South-Western Pub.

Naldi, L., Nordqvist, M., Sjöberg, K., and Wiklund, J. (2007). Entrepreneurial orientation, risk taking, and performance in family firms. Family Business Review, 20(1), 33-47.

Naman, J. L., \& Slevin, D. P. (1993). Entrepreneurship and the concept of fit: A model and empirical tests. Strategic Management Journal, 14(2), 137-153.

Nordqvist, M., Habbershon, T. G., and Melin, L. (2008). Transgenerational entrepreneurship: Exploring entrepreneurial orientation in family firms. In H. Landstro"m, D. Smallbone, H. Crijns, and E. Laveren (Eds.), Entrepreneurship, sustainable growth and performance: Frontiers in European entrepreneurship research (pp. 93-116). London: Edward Elgar.

Rauch, A., Wiklund, J., Lumpkin, G., and Frese, M. (2009). Entrepreneurial orientation and business 
performance: An assessment of past research and suggestions for the future. Entrepreneurship Theory and Practice, 33(3), 761.

Runyan, R. C., Ge, B., Dong, B., \& Swinney, J. L. (2012). Entrepreneurial orientation in Cross Cultural research: Assessing measurement invariance in the construct. Entrepreneurship Theory and Practice, 36 (4), 819-836.

Schollhammer, H. (1982). Internal corporate entrepreneurship. In C. A. Kent, D. L. Sexton, and K. H. Vesper (Eds.), Encyclopedia of entrepreneurship, pp. 209-223. Englewood Cliffs, NJ: Prentice Hall.

Schulze, W.S., Lubatkin, M.H., Dino, R. N., and Buchholtz, A.K. (2001). Agency relationships in family firms: Theory and evidence. Organization Science, 12(2): 99-116.

Schulze, W., Lubatkin, M., and Dino, R. (2003). Toward a theory of agency and altruism in family firms. Journal of Business Venturing, 18, 473-490.

Short, J.C., Broberg, J.C., Cogliser, C.C., and Brigham, K.H. (2009). Construct validation using computer-aided text analysis (CATA): An illustration using entrepreneurial orientation. Organizational Research Methods, 12, 1-28.

Stam, W., \& Elfring, T. (2008). Entrepreneurial orientation and new venture performance: The moderating role of intra-and extraindustry social capital. The Academy of Management Journal ARCHIVE, 51(1), 97-111.

Stevenson, H. H., \& Jarillo, J. C. (2007). A paradigm of entrepreneurship: Entrepreneurial management. Harvard Business School, , 155-170.

Tang, J., Tang, Z., Marino, L.D., Zhang, Y., and Li, Q. (2008). Exploring an inverted u-shape relationship between entrepreneurial orientation and performance in Chinese ventures. Entrepreneurship Theory and Practice, 32, 219-239.

Tang, J. and Hull, C. (2012). An investigation of EO, perceived environmental hostility, and strategy application among chinese SMEs. Journal of Small Business Management, 50 (1), 132-158.

Ventakraman, N. (1989) "Strategic orientation of business enterprises: the construct, dimensionality, and measurement". Management Science, 35:423-44

Wang, C. L. (2008). Entrepreneurial orientation, learning orientation, and firm performance.
Entrepreneurship Theory and Practice, 32(4), 635657.

Weismeier-Sammer, D. (2011). Entrepreneurial behavior in family firms: A replication study. Journal of Family Business Strategy, 2(3), 128-138.

Wiklund, J., and Shepherd, D. (2005). Entrepreneurial orientation and small business performance: A configurational approach. Journal of Business Venturing, 20, 71-91.

Yin, R.K. (1994), Case Study Research. Design and Methods (2a ed.), London: Sage Publications.

Zahra, S. (1991). Predictors and financial outcomes of corporate entrepreneurship: An exploratory study. Journal of Business Venturing, 6, 259-285.

Zahra, S. A., \& Covin, J. G. (1993). Business strategy, technology policy and firm performance. Strategic Management Journal, 14(6), 451-478.

Zahra, S. and Covin, G. (1995) Contextual influences on the CE-performance relationship: a longitudinal analysis. Journal of Business Venturing, 10 (1), 43-58.

Zahra, S. and Neubaum, D. (1998) Environmental adversity and the entrepreneurial activities of new ventures. Journal of Developmental Entrepreneurship, 3.

Zahra, S., and Garvin, D. (2000). International corporate entrepreneurship and firm performance: The moderating effects of international environment hostility. Entrepreneurship Theory and Practice, 15(4), 469-492.

Zahra, S. A. (2005). Entrepreneurial risk taking in family firms. Family Business Review, 18(1), 2340.

Zellweger, T.W. and Sieger, P. (2010). Entrepreneurial orientation in long-lived family firms, Small Business Economics, DOI 10.1007/s11187-010-9267-6

Zellweger, T.M., Kellermanns; F.W., and Eddleston; K.A. (2008). Building a family firm image: How family firms capitalize on their families. Paper presented at the USASBE. 